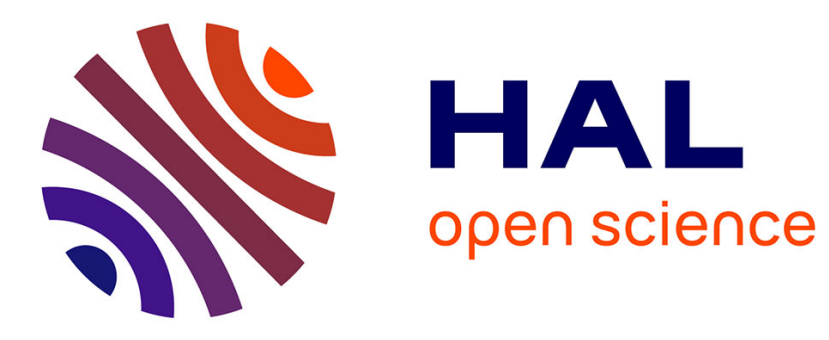

\title{
Zoning-based Localization in Indoor Sensor Networks Using Belief Functions Theory
}

\author{
Daniel Alshamaa, Farah Mourad-Chehade, Paul Honeine
}

\section{To cite this version:}

Daniel Alshamaa, Farah Mourad-Chehade, Paul Honeine. Zoning-based Localization in Indoor Sensor Networks Using Belief Functions Theory. Proc. 17th IEEE International Workshop on Signal Processing Advances in Wireless Communications (SPAWC), 2016, Edinburgh, UK, Unknown Region. 10.1109/SPAWC.2016.7536787 . hal-01965915

\section{HAL Id: hal-01965915 https://hal.science/hal-01965915}

Submitted on 27 Dec 2018

HAL is a multi-disciplinary open access archive for the deposit and dissemination of scientific research documents, whether they are published or not. The documents may come from teaching and research institutions in France or abroad, or from public or private research centers.
L'archive ouverte pluridisciplinaire HAL, est destinée au dépôt et à la diffusion de documents scientifiques de niveau recherche, publiés ou non, émanant des établissements d'enseignement et de recherche français ou étrangers, des laboratoires publics ou privés. 


\section{Zoning-based Localization in Indoor Sensor Networks Using Belief Functions Theory}

\author{
Daniel AlShamaa, Farah Mourad-Chehade \\ Institut Charles Delaunay, ROSAS, LM2S \\ Université de Technologie de Troyes \\ UMR 6281, CNRS, Troyes, France \\ daniel.alshamaa@utt.fr, farah.chehade@utt.fr
}

\author{
Paul Honeine \\ LITIS lab \\ Université de Rouen \\ Rouen, France \\ paul.honeine@univ-rouen.fr
}

\begin{abstract}
Localization is an essential issue in wireless sensor networks to process the information retrieved by sensor nodes. This paper presents an indoor zoning-based localization technique that works efficiently in real environments. The targeted area is composed of several zones, the objective being to find the zone where the mobile node is instantly located. The proposed approach collects first strengths of received WiFi signals from neighboring access points and builds a fingerprints database. It then uses belief functions theory to combine all measured data and define an evidence framework, to be used afterwards for estimating the most probable node's zone. Real experiments demonstrate the effectiveness of this approach and its competence compared to state-of-the-art methods.
\end{abstract}

Index Terms-Belief functions, indoor environment, radio fingerprints, wireless sensor networks, zoning.

\section{INTRODUCTION}

Massive advances in wireless communications and electronics have fomented the development of heavily distributed wireless sensor networks constituted of hundreds of sensor nodes. These networks help in performing several tasks ranging from medical and military applications, to monitoring homes, hospitals and forests [1]. This paper considers in particular the zoning-based localization problem in indoor environments, that aims to find the zone where a sensor node resides, instead of its exact position. This issue is important for health-care applications for instance, where Alzheimer's patients might be lost in their nursing home [2] and where locating them in a specific zone is completely sufficient.

A first solution for zoning-based localization is to find exact locations of sensors and then determine their zones using their estimated locations. Many existing works have considered the exact localization problem. Indeed, the integration of a GPSGSM system into sensor nodes is widely used in outdoor tracking systems [3]. However, it has limitations in indoor environments [4]. Alternative solutions consist of using other types of signals, that are available in closed environments, like ultra wide band, WiFi, zigbee, bluetooth,etc [5], [6], [7], [8]. One of the advantages of WiFi signals over the others is that one can rely only on the Access Points (APs) present inside the building, with no additional hardware. The localization issue consists then on finding the location of a sensor node according to the WiFi signals that it collects from APs. Several localization algorithms using strengths of WiFi signals were developed. Trilateration and connectivity- based methods have been developped for localization using the pathloss model, which is not efficient especially in indoor environments [9], [10], [11], [12]. Alternatively, techniques that employ fingerprinting are widely implemented. They consist of collecting a database of exact reference locations, coupled to their corresponding WiFi signals strengths, received from the APs. The localization problem is then solved using this database and the k-nearest neighbors scheme [13], neural networks [14], [15] or kernel-based learning [16], [17].

The disadvantage of exact localization algorithms using fingerprinting is their need to a database with exact locations, whose construction is time-costly and complex. Some researchers have tackled the zoning problem without computing exact locations [18], [19]. In [18], the signal strengths are periodically collected while the user is moving. When a sharp change in the strength indicator is detected, the zone is determined based on the user's path. On the other hand, the design of overlapping zone partitioning is implemented in [19]. Alternatively, fingerprints of WiFi strengths and zones numbers could be collected. Here the zoning problem could be addressed as a multiclass classification issue, resolved using for instance support vector machines [20] or the logistic regression [21].

This paper proposes an original zoning-based localization technique that makes use of belief functions to combine evidence revealed at each sensor. At a preliminary phase, the proposed method consists of constructing a fingerprinting database that associates to each zone a set of WiFi signals strengths collected from the APs. Each AP is then considered as a source of information and is used in the belief functions framework to set a mass function over the zones. Afterwards, once the mobile node is in an unknown zone, it measures its signal strengths and use the constructed mass functions to estimate the zone where it resides the most probably. One advantage of this method is that it yields a set of possible solutions, sorted in a descent order of priority. Real experiments are conducted in the first floor of a three floors building. Results show the effectiveness of the proposed method, compared to other state-of-the-art algorithms.

The paper is organized as follows. Section II briefly introduces the belief functions theory. Section III describes the proposed method. Section IV presents the experimental setup and results, while Section V concludes the paper. 


\section{BELIEF FunCTIONS THEORY}

The Belief Functions Theory (BFT) is also known as Dempster-Shafer or evidence theory. It is a branch of mathematics that provides an original framework for data fusion [22]. In the following, the basics of the BFT are introduced first. The main concepts of data combination and decision making are given next.

\section{A. Basic definitions}

Let $\theta$ be a discrete variable, taking values in $\Theta=$ $\left\{\theta_{1}, \ldots, \theta_{n}\right\}$ and let $2^{\Theta}$ be the set of all the subsets of $\Theta$, i.e. $2^{\Theta}=\left\{\emptyset,\left\{\theta_{1}\right\}, \ldots, \Theta\right\}$. One fundamental function of the BFT is the mass function, also called the basic belief assignment. A mass function $m_{S}($.$) is a mapping from 2^{\Theta}$ to the interval $[0,1]$, defined according to a certain information source $S$. It satisfies:

$$
\sum_{A \in 2^{\ominus}} m_{S}(A)=1 .
$$

The mass $m_{S}(A)$ given to $A \in 2^{\Theta}$ stands for the proportion of evidence, brought by the source $S$, saying that the observed variable $\theta$ belongs to $A$.

Another important notion of the BFT is the belief function, denoted by bel $l_{S}($.$) and derived from the mass function. It is$ defined from $2^{\Theta}$ into $[0,1]$ as follows:

$$
\operatorname{bel}_{S}(A)=\sum_{\substack{A_{i} \subseteq A \\ A_{i} \neq \emptyset}} m_{S}\left(A_{i}\right) .
$$

The plausibility function, $p l_{S}: 2^{\theta} \rightarrow[0,1]$, is also a notion of the BFT, derived from the mass function as follows:

$$
p l_{S}(A)=\sum_{A_{i} \cap A \neq \emptyset} m_{S}\left(A_{i}\right) .
$$

The belief of $A$ can be interpreted as the proportion of available evidence given to $A$, while its plausibility is a measure of the maximum support of evidence that could be given to $A$ [22]. The probability to have $\theta \in A$ falls within an interval whose lower and higher bounds are given by the belief and plausibility values respectively [23]. In general, there is no unified framework for mass construction. Any algorithm that tansforms observations into mass functions while satisfying equation (1) can be adopted. Moreover, Shafer shows that, given any one of $m($.$) , \operatorname{bel}($.$) , or p l($.$) , the others can be$ derived [24].

\section{B. Evidence combination and decision}

Let $S_{1}$ and $S_{2}$ be two sources of information about the variable $\theta$. According to the sources' information, two mass functions could be defined $m_{S_{1}}($.$) and m_{S_{2}}($.$) , or simply$ $m_{1}($.$) and m_{2}($.$) respectively. Combining the evidence con-$ sists of aggregating the information coming from the two sources $S_{1}$ and $S_{2}$. Different combination rules exist in literature, such as the normalized conjunctive rule [22]. Also called the Dempster's combinational rule, the normalized conjunctive rule leads to a more informative and specialized mass function.
According to this rule, the two mass functions $m_{1}($.$) and m_{2}($. are combined first into a unified one $m_{1 \cap 2}($.$) as follows,$

$$
m_{1 \cap 2}(A)=\sum_{A_{i} \cap A_{j}=A} m_{1}\left(A_{i}\right) m_{2}\left(A_{j}\right),
$$

for all the subsets $A \in 2^{\Theta}$. By applying equation (4), $\emptyset$ might have a certain mass $m_{1 \cap 2}(\emptyset) \neq 0$, representing the conflict of both sources. This computation is then followed by a normalization phase leading to one final mass function $m_{1 \oplus 2}($.$) given by,$

$$
m_{1 \oplus 2}(A)=\frac{m_{1 \cap 2}(A)}{1-m_{1 \cap 2}(\emptyset)}, \text { for } A \neq \emptyset,
$$

with $m_{1 \oplus_{2}}(\emptyset)=0$. This combination rule could be easily extended to a higher number of sources. Once $m_{1} \oplus_{2}($.$) is$ constructed, the decision rule consists of selecting the subset $A \in 2^{\Theta}$ having the highest mass. Alternatively, one can choose the subset having the highest plausibility, the highest pignistic probability, etc [24].

\section{Zoning-BASED LOCALIZATION METHOD}

This section presents the employed method targeting the localization problem in indoor environments. Consider a building is divided into $N_{Z}$ zones denoted by $Z_{k}, k=$ $1,2, \ldots, N_{Z}$. Having a mobile node moving within these zones, the objective of the method is to find instantly the zone where it is accurately. To this end, the node uses the WiFi signals it collects from the Access Points (APs) neighboring it, in the belief functions framework.

\section{A. Description of the method}

Let $N_{A P}$ be the number of APs in the area of interest, denoted by $A P_{n}, n=1,2 \ldots, N_{A P}$. The APs broadcast WiFi signals in the network. The mobile node, free to move between the zones, collects instantly the APs signals and measures the Received Signal Strength Indicators (RSSIs) with respect to every $A P_{n}$, denoted by $\rho_{n}(t)$, where $t$ is the time instant. The objective is to affiliate the mobile node to its corresponding zone $\hat{Z}(t)$ based on its online RSSI measurements $\rho_{n}(t), n=1,2, \ldots, N_{A P}$. This is done using the fingerprinting technique and the belief functions theory through three phases: constructing radio-fingerprints, computing the mass functions, and then estimating the zone by decision fusion in a real time process. As for the database construction, a mobile node, moves randomly in each zone of the targeted area in a preliminary phase and measures the RSSIs of WiFi signals from all APs. Suppose $\rho_{k, n, \ell}$ corresponds to the $\ell$-th RSSI measured inside zone $Z_{k}$ with respect to $A P_{n}$. Let $N_{k}$ be the number of RSSIs measurements done in a zone $Z_{k}$. This implies that for a certain zone and a given AP, a set of $N_{k}$ values is collected representing the variations of the RSSI in this zone with respect to that AP. Let $N_{D}=\sum_{k=1}^{N_{Z}} N_{k}$ be the total number of measurements. Then a database of $N_{D} \times N_{A P}$ RSSIs labeled to the zones is then obtained. This database is used in the belief functions framework with the instant RSSIs $\rho_{n}(t)$ of a mobile node to select its zone, as is shown in the following. 


\section{B. Computation of mass functions}

The zoning-based localization problem is solved using the belief functions theory. To this end, before moving to online computations, mass functions should be constructed, in a way to transform the APs information, ie. RSSIs, into masses. One of many ways to do that is to represent each set of RSSI values of the database as a Gaussian distribution per zone per AP. In this way, one obtains $N_{Z} \times N_{A P}$ Gaussian functions. Let $\mu_{k, n}$ and $\sigma_{k, n}$ be the respective average and standard deviation of the RSSIs of zone $Z_{k}$ with respect to $A P_{n}$, that is,

$$
\mu_{k, n}=\frac{1}{N_{k}} \sum_{\ell=1}^{N_{k}} \rho_{k, n, \ell} \text { and } \sigma_{k, n}=\sqrt{\frac{1}{N_{k}} \sum_{\ell=1}^{N_{k}} \rho_{k, n, \ell}^{2}-\mu_{k, n}^{2}}
$$

with $k=1,2, \ldots, N_{Z}$ and $n=1,2, \ldots, N_{A P}$. Having now the online RSSI measurement $\rho_{n}(t)$ of the considered mobile node with respect to $A P_{n}, n=1,2, \ldots, N_{A P}$, the probability of the node to be in zone $Z_{k}$ according to $A P_{n}$ is computed by applying the corresponding Gaussian function to $\rho_{n}(t)$, i.e.,

$$
P_{k, n}\left(\rho_{n}(t)\right)=\frac{1}{\sigma_{k, n} \sqrt{2 \pi}} \exp \left(-\frac{\left(\rho_{n}(t)-\mu_{k, n}\right)^{2}}{2 \sigma_{k, n}^{2}}\right) .
$$

The amount of belief that the mobile node, measuring $\rho_{n}(t)$, belongs to zone $Z_{k}$, according to the information source $A P_{n}$ is given by:

$$
m_{n, t}\left(Z_{k}\right)=\frac{P_{k, n}\left(\rho_{n}(t)\right)}{\sum_{v=1}^{N_{Z}} P_{v, n}\left(\rho_{n}(t)\right)} .
$$

Due to this definition, one obtains a normalized mass function that attributes the highest mass to the zone whose collected RSSIs are the closest to the online RSSI $\rho_{n}(t)$ of the node.

\section{Zone estimation}

The $N_{A P}$ mass functions (8) are combined into one unified mass function using the conjunctive rule of combination,

$$
m_{\cap, t}\left(Z_{k}\right)=\prod_{n=1}^{N_{A P}} m_{n}\left(Z_{k}\right) .
$$

This unified mass function undergoes a normalization phase to acquire one final mass function, as follows,

$$
m_{\oplus, t}\left(Z_{k}\right)=\frac{m_{\cap, t}\left(Z_{k}\right)}{1-m_{\cap, t}(\emptyset)} .
$$

The fusion of evidence allows to associate the mobile node with a certain amount of belief to each zone. The decision rule to be adopted consists then of selecting the zone $\hat{Z}(t)$ that has the highest mass,

$$
\hat{Z}(t)=\arg \max _{k=1 \ldots N_{Z}} m_{\oplus, t}\left(Z_{k}\right) .
$$

One advantage of the proposed method remains in the way that estimation stays possible even if one AP is not detected by the node. Indeed, in this case, its mass is not considered in the computation of the conjunctive mass in equation (9). Another advantage is that several zones are obtained with a certain amount of belief, which makes possible to select a second-choice zone, having the second highest mass, in case the first one is incorrect.

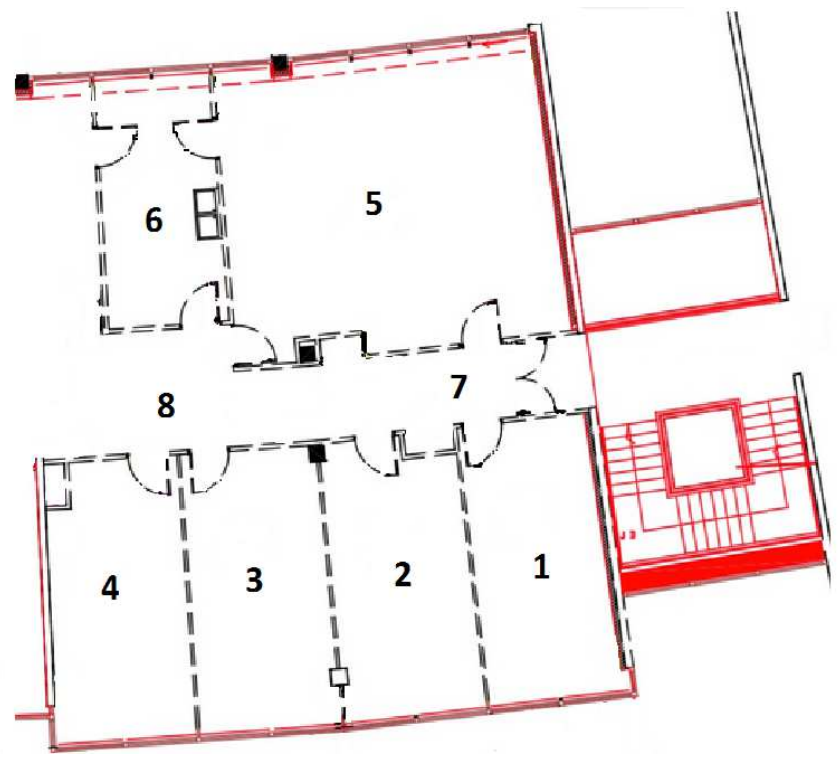

Fig. 1: A sector of the first floor of the statistical and operational research department of the University of Technology of Troyes

\section{EXPERIMENTS}

To evaluate the performance of the proposed method, real experiments are conducted in a sector of the University of Technology of Troyes, France. In the following, experimental setups are first introduced. The illustration of the proposed method and its comparison to state-of-the-art methods are shown afterwards.

\section{A. Experimental setups}

This paper considers real experiments that are realized in a WLAN environment at the first floor of the statistical and operational research department of the University of Technology of Troyes, France. As shown in Fig. 1, the considered sector of approximated area of $190 \mathrm{~m}^{2}$ is partitioned into six offices, from both sides of a corridor, that we divided into two zones, according to its architecture. This leads to eight zones in the considered area. A personal computer, with a WiFi scanner software ${ }^{1}$, can distinguish APs of the network throughout their MAC addresses. It measures then the RSSIs of their transmitted signals. Note that several APs could be detected at the considered area. Only the APs located in the ground, first and second floors of the building to which belongs the studied area are considered. This leads to six of the total APs installed in the network. A set of 30 measures are taken in each zone, of which some are randomly used to construct the databases, and the others are kept for test and validation. The measures are taken in random positions and orientation of the personal computer.

\footnotetext{
${ }^{1}$ Wi-Fi Scanner by Lizard Systems
} 
TABLE I: Number of incorrect estimated zones with $30 \%$ test points

\begin{tabular}{|c|c|c|}
\hline Zone number & 1st choice & 2nd choice \\
\hline $\mathbf{1}$ & 0 & - \\
\hline $\mathbf{2}$ & 1 & 0 \\
\hline $\mathbf{3}$ & 1 & 0 \\
\hline $\mathbf{4}$ & 0 & - \\
\hline $\mathbf{5}$ & 0 & - \\
\hline $\mathbf{6}$ & 1 & - \\
\hline $\mathbf{7}$ & 1 & 0 \\
\hline $\mathbf{8}$ & 0 & - \\
\hline Total & 4 & 0 \\
\hline
\end{tabular}

\section{B. Illustration of the proposed method}

In order to illustrate the proposed method, $70 \%$ of the collected RSSIs are first randomly selected at each zone for the database, that is 21 measures, keeping 9 for testing per zone. Then, the RSSIs database is used to compute, Gaussian density functions, which leads to six graphs, each corresponding to an AP. Within each graph, eight Gaussian functions represent the variations of RSSIs in the eight zones. Fig. 2 displays these variations with respect to the first AP. As clearly indicates this figure, the partial overlapping between the Gaussian functions is wide and hence, the zones' masses can be easily miscalculated considering one AP. However, when considering all the APs and applying the belief functions to combine evidence, we acquire interesting results, which is shown in the following.

Having $70 \%$ of the collected RSSIs in the database, we test our method localizing the $30 \%$ remaining measurements, that is 9 test points per zone. Table I shows the numbers of incorrect estimated zones if only the zone having the highest mass (1st choice) is considered and also in case the zone having the second highest mass (2nd choice) is considered as well. The table shows also the total number of erroneous estimations over the 72 total test points in both cases. The results show clearly that the proposed method performs well, with only $5.55 \%$ of errors if the first choice is only considered, which is totally corrected if the second choice zone is also selected. Moreover, in order to measure the performance of the method under low data availability, the ratio of the measurements used to construct the database is reduced to $60 \%$ then to $50 \%$, while testing over the other $40 \%$ and $50 \%$ respectively. The total numbers of erroneous estimated zones are shown in Table II. As expected, the percentage of erroneous estimations increases with the 1 st choice zone when the constructed database is reduced to $60 \%$ then to $50 \%$. However, it is yet considered to be low with $7.29 \%$ in the first case and $8.33 \%$ in the second. It is noticed that 1 out of the 120 measurements was not recovered by the second choice, rather by the third one. This erroneous point, located in zone 7 , was estimated to be in zone 5 then in zone 2 . This is due to the insufficient built database which made zone 7 incapable of representing its variations in front of the neighboring zones in the best possible way.

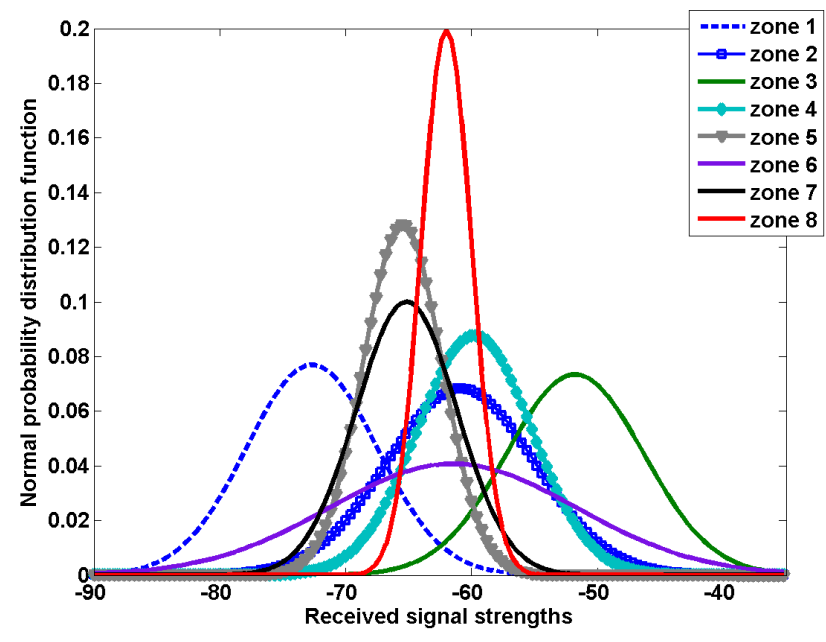

Fig. 2: Gaussian functions of the eight zones with respect to the first AP

TABLE II: Number of incorrect estimated zones with low data availability, for three different ratios of database-test

\begin{tabular}{|l|l|c|}
\cline { 3 - 3 } \multicolumn{2}{c|}{} & Number of errors / total test points \\
\hline \multirow{2}{*}{$\mathbf{7 0 \% - 3 0 \%}$} & 1st choice & $4 / 72$ \\
\cline { 2 - 3 } & 2nd choice & $0 / 72$ \\
\hline \multirow{2}{*}{$\mathbf{6 0 \% - 4 0 \%}$} & 1st choice & $7 / 96$ \\
\cline { 2 - 3 } & 2nd choice & $0 / 96$ \\
\hline \multirow{2}{*}{$\mathbf{5 0 \% - 5 0 \%}$} & 1st choice & $10 / 120$ \\
\cline { 2 - 3 } & 2nd choice & $1 / 120$ \\
\hline
\end{tabular}

\section{Comparison to state-of-the-art methods}

In this section, the proposed method is compared to both the Multinominal Logistic Regression (MLR) and Support Vector Machines (SVM) methods. MLR is a natural extension of binary logistic regression to multiclass classification problems [21], [25]. It uses maximum likelihood estimation to evaluate the probability of the node being in each zone. MLR leads then to a first choice and a second choice zones, as well. As for SVM, it is among the best supervised learning algorithms. It is based on statistical learning theory to determine the location of decision boundaries that produces the optimal separation of the classes [26]. The one against one strategy of SVM is adopted in this paper, especially that the the number of classes is not too big, and it is suitable with kernel algorithms. Only one estimated zone is then obtained using SVM. Table III shows the percentage of errors over the test points obtained with our proposed method (BFT) compared to both MLR and SVM for the three sizes of the databases $70 \%, 60 \%$ and $50 \%$ of the available data. The results show that our proposed method outperforms the other methods, even with less computations. 
TABLE III: Comparison of BFT method to MLR and SVM methods, in terms of percentage of error

\begin{tabular}{|c|c|c|c|c|}
\cline { 3 - 5 } \multicolumn{2}{c|}{} & \multicolumn{3}{c|}{ Ratio of database-test } \\
\cline { 3 - 5 } \multicolumn{2}{c|}{} & $\mathbf{7 0 \% - 3 0 \%}$ & $\mathbf{6 0 \% - 4 0 \%}$ & $\mathbf{5 0 \% - 5 0 \%}$ \\
\hline \multirow{2}{*}{ BFT } & 1st choice & 5.55 & 7.29 & 8.33 \\
\cline { 2 - 5 } & 2nd choice & 0 & 0 & 0.83 \\
\hline \multirow{2}{*}{ MLR } & 1st choice & 16.66 & 19.79 & 20.83 \\
\cline { 2 - 5 } & 2nd choice & 5.55 & 5.21 & 7.5 \\
\hline \multirow{2}{*}{ SVM } & 13.89 & 16.66 & 17.5 \\
\hline
\end{tabular}

\section{CONCLUSION}

This paper presented a zoning-based localization technique for indoor environments using belief functions theory. The proposed method constructs a RSSI database using Access Points WiFi signals in an offline phase. It then computes mass functions that associate to each zone certain amounts of evidence. Combining these masses, together with the mobile node online RSSIs, using the belief functions theory, allows to determine the correct zone where it is located. Real experimental results showed the effectiveness of the proposed method, outperforming the multinominal logistic regression and support vector machines techniques. Future works will deal with multi-floor buildings and Access Points failure problems. The mobility of sensor nodes would also be used to improve the first choice zone estimation.

\section{ACKNOWLEDGEMENT}

The authors would like to thank the Champagne-Ardenne Region in France for funding this work, project LIPAD.

\section{REFERENCES}

[1] G. Mao, B. Fidan, and B. D. Anderson, "Wireless sensor network localization techniques," Computer Networks, vol. 51, no. 10, pp. 2529 - 2553, 2007.

[2] Y.-C. Liu, Y.-K. Ou, S.-N. Lin, and C.-W. Fang, "A study of the indoor walking navigation system for patients with early-stage Alzheimers disease," in International Conference on Computer, Networks and Communication Engineering (ICCNCE 2013), Atlantis Press, 2013.

[3] M. A. Al-Khedher, "Hybrid GPS-GSM localization of automobile tracking system," CoRR, vol. abs/1201.2630, 2012.

[4] T. Oshin, S. Poslad, and A. Ma, "Improving the energy-efficiency of GPS based location sensing smartphone applications," in Trust, Security and Privacy in Computing and Communications (TrustCom), 2012 IEEE 11th International Conference on, pp. 1698-1705, June 2012.

[5] H. Liu, H. Darabi, P. Banerjee, and J. Liu, "Survey of wireless indoor positioning techniques and systems," Systems, Man, and Cybernetics, Part C: Applications and Reviews, IEEE Transactions on, vol. 37, pp. 1067-1080, Nov 2007.

[6] H.-S. Ahn and W. Yu, Indoor localization techniques based on wireless sensor networks. INTECH Open Access Publisher, 2009.

[7] A. Disha, "A comparative analysis on indoor positioning techniques and systems," International Journal of Engineering Research and Applications, vol. 3, pp. 1790-1796, 2013.

[8] A. Bekkelien, M. Deriaz, and S. Marchand-Maillet, "Bluetooth indoor positioning," Master's thesis, University of Geneva, 2012.

[9] J. Sangthong, P. Dokpikul, and S. Promwong, "Indoor positioning based on IEEE 802.15. 4a standard using trilateration technique and UWB signal," in Proceedings in progress in electromagnetics research symposium, 2012.
[10] M. Aruna, R. Ganesan, and A. Renold, "Optimized path planning mechanism for localization in wireless sensor networks," in Smart Technologies and Management for Computing, Communication, Controls, Energy and Materials (ICSTM), 2015 International Conference on, pp. 171-177, May 2015.

[11] J. M. Pak, C. K. Ahn, Y. Shmaliy, and M. T. Lim, "Improving reliability of particle filter-based localization in wireless sensor networks via hybrid particle/FIR filtering," Industrial Informatics, IEEE Transactions on, vol. 11, pp. 1089-1098, Oct 2015.

[12] F. Mourad, H. Snoussi, F. Abdallah, and C. Richard, "Anchor-based localization via interval analysis for mobile ad-hoc sensor networks," Signal Processing, IEEE Transactions on, vol. 57, no. 8, pp. 3226-3239, 2009.

[13] D. Liang, Z. Zhang, A. Piao, and S. Zhang, "Indoor localization algorithm based on iterative grid clustering and AP scoring," in Personal, Indoor, and Mobile Radio Communications (PIMRC), 2015 IEEE 26th Annual International Symposium on, pp. 1997-2001, IEEE, 2015.

[14] S. Tuncer and T. Tuncer, "Indoor localization with bluetooth technology using artificial neural networks," in Intelligent Engineering Systems (INES), 2015 IEEE 19th International Conference on, pp. 213-217, IEEE, 2015.

[15] S.-H. Fang and T.-N. Lin, "Indoor location system based on discriminant-adaptive neural network in IEEE 802.11 environments," Neural Networks, IEEE Transactions on, vol. 19, no. 11, pp. 1973-1978, 2008.

[16] S. Mahfouz, F. Mourad-Chehade, P. Honeine, J. Farah, and H. Snoussi, "Target tracking using machine learning and kalman filter in wireless sensor networks," Sensors Journal, IEEE, vol. 14, pp. 3715-3725, Oct 2014.

[17] C. Figuera, J. L. Rojo-Álvarez, M. Wilby, I. Mora-Jiménez, and A. J. Caamaño, "Advanced support vector machines for 802.11 indoor location," Signal Processing, vol. 92, no. 9, pp. 2126-2136, 2012.

[18] D. L. Lee and Q. Chen, "A model-based WiFi localization method," in Proceedings of the 2nd international conference on Scalable information systems, p. 40, ICST (Institute for Computer Sciences, SocialInformatics and Telecommunications Engineering), 2007.

[19] K. K. Khedo, D. Sathan, R. Elaheebocus, R. K. Subramanian, and S. Rughooputh, "Overlapping zone partitioning localisation technique for RFID," International Journal of Ubiquitous Computing (IJU), vol. 1, no. 2, pp. 20-32, 2010.

[20] S. Karamizadeh, S. M. Abdullah, M. Halimi, J. Shayan, and M. J. Rajabi, "Advantage and drawback of support vector machine functionality," in Computer, Communications, and Control Technology (I4CT), 2014 International Conference on, pp. 63-65, IEEE, 2014.

[21] K. Coussement, F. A. V. den Bossche, and K. W. D. Bock, "Data accuracy's impact on segmentation performance: Benchmarking $\{\mathrm{RFM}\}$ analysis, logistic regression, and decision trees," Journal of Business Research, vol. 67, no. 1, pp. 2751 - 2758, 2014.

[22] W. Zhang and Z. Zhang, "Belief function based decision fusion for decentralized target classification in wireless sensor networks," Sensors, vol. 15, no. 8, pp. 20524-20540, 2015.

[23] J. J. Sudano, "Pignistic probability transforms for mixes of low- and high-probability events," CoRR, vol. abs/1505.07751, 2015.

[24] G. Shafer, A mathematical theory of evidence. Princeton university press, 1976.

[25] G. Mauša, T. G. Grbac, and B. D. Bašić, "Multivariate logistic regression prediction of fault-proneness in software modules," in MIPRO, 2012 Proceedings of the 35th International Convention, pp. 698-703, IEEE, 2012.

[26] M. Pal and G. M. Foody, "Evaluation of SVM, RVM and SMLR for accurate image classification with limited ground data," Selected Topics in Applied Earth Observations and Remote Sensing, IEEE Journal of, vol. 5, no. 5, pp. 1344-1355, 2012. 